ARQGA / 1070

\title{
COMPARAÇÃO ENTRE ACALÁSIA IDIOPÁTICA E ACALÁSIA CONSEQÜENTE À DOENÇA DE CHAGAS: revisão de publicações sobre o tema
}

\author{
Roberto Oliveira DANTAS*
}

RESUMO - Racional - Embora acalásia idiopática e acalásia conseqüente à doença de Chagas tenham manifestações clínicas semelhantes, mesmo tratamento e ambas comprometerem o plexo mientérico do esôfago, é possível que as alterações motoras do esôfago provocadas pelas duas doenças não sejam iguais, conseqüência da diferente intensidade da destruição dos neurônios inibitórios e excitatórios do esôfago. Objetivos - Revisar os trabalhos que estudaram a fisiopatologia e as alterações motoras do esôfago na acalásia idiopática e na doença de Chagas. Dados e fontes de referências - Foram revistos trabalhos que definiram as características da acalásia idiopática e aquela provocada pela doença de Chagas. Síntese dos dados - Está demonstrado o comprometimento da inervação inibitória do esôfago nas duas doenças. Em relação à inervação excitatória, os resultados dos estudos dos efeitos do edrofônio, da atropina e da toxina botulínica sugerem que ela está mais comprometida na doença de Chagas do que na acalásia idiopática, o que justificaria a maior pressão do esfíncter inferior do esôfago observada na acalásia idiopática. Os pacientes com doença de Chagas têm mais falhas de contrações e maior freqüência de anticorpos contra os receptores muscarínicos M2 da acetilcolina. A duração da contração em corpo esofágico é maior nos pacientes com acalásia

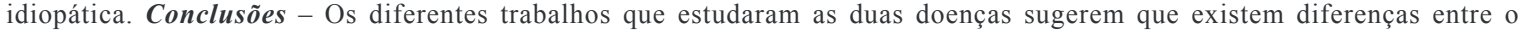
comprometimento do esôfago na acalásia idiopática e na doença de Chagas, principalmente no comprometimento da inervação excitatória, mais intenso na doença de Chagas.

DESCRITORES - Acalásia esofágica. Doença de Chagas. Transtornos da motilidade esofágica.

Acalásia é a mais conhecida doença motora do esôfago, caracterizada por relaxamento parcial ou ausente do esfíncter inferior do esôfago (EIE) e contrações não-peristálticas no corpo esofágico ${ }^{(44)}$. No exame radiológico é encontrada retenção do meio de contraste no esôfago, incoordenação no trânsito, afilamento regular da transição esofagogástrica e esôfago dilatado. O sintoma mais freqüente é a disfagia, seguida pela regurgitação, pirose e dor torácica ${ }^{(44)}$.

A doença primária, quando compromete somente o esôfago, não tem etiologia conhecida, sendo infecção por vírus a hipótese mais provável ${ }^{(20,27)}$. A doença secundária, quando doença sistêmica provoca a alteração motora do esôfago, tem grande número de causas $^{(20,27)}$. No Brasil a doença de Chagas é a mais importante ${ }^{(36,37)}$.
Acalásia idiopática é doença pouco freqüente, com prevalência estimada de 7 a 13 casos por 100.000 habitantes ${ }^{(20,35)}$. Acalásia provocada pela doença de Chagas afeta de $7 \%$ a $10 \%$ das pessoas infectadas pelo Trypanosoma cruzi $^{(37)}$. Durante muitos anos o número de pessoas no Brasil com doença de Chagas foi muito grande e, como conseqüência, a prevalência de megaesôfago no Brasil foi muito maior do que a observada em outros países. O combate intensivo visando diminuir a transmissão da doença ocorrido na segunda metade do século XX, fez com que a prevalência diminuísse rapidamente, estando atualmente restrita a casos crônicos e a algumas regiões do país.

O termo acalásia é referente à doença motora do esôfago e megaesôfago a sua conseqüência. Embora haja muita semelhança

\footnotetext{
* Departamento de Clínica Médica da Faculdade de Medicina de Ribeirão Preto da Universidade de São Paulo, Ribeirão Preto, SP.

Endereço para correspondência: Dr. Roberto Oliveira Dantas - Departamento de Clínica Médica - Faculdade de Medicina de Ribeirão Preto -

Universidade de São Paulo - 14049-900 - Ribeirão Preto, SP. e-mail: rodantas@fmrp.usp.br
} 
entre o megaesôfago idiopático e aquele provocado por doença de Chagas, têm sido observadas algumas diferenças entre elas ${ }^{(8)}$. Não é surpresa que tal fato ocorra, pois, entre as várias doenças que podem provocar acalásia, existem diferentes graus de lesão no esôfago ${ }^{(27)}$.

Nesta revisão foram incluídos trabalhos que estudaram as características do envolvimento do esôfago na acalásia idiopática e na acalásia conseqüente à doença de Chagas, principalmente aqueles que compararam as duas doenças utilizando o mesmo método.

De comum entre acalásia idiopática e chagásica há a observação de perda dos neurônios do plexo mientérico do esôfago ${ }^{(23,30)}$. Entretanto, estudos da fisiopatologia da acalásia descrevem que a lesão do plexo mientérico ocorre mais intensamente nos neurônios inibitórios, sendo os neurônios excitatórios lesados em graus variáveis de intensidade ${ }^{(27)}$, o que provoca variações na apresentação da doença motora do esôfago ${ }^{(28)}$. A intensidade do comprometimento do plexo mientérico provoca grande variação na esofagopatia conseqüente à doença de Chagas $^{(11,12,36)}$. Os trabalhos que tiveram por objetivo o estudo da fisiopatologia e alterações manométricas observadas no megaesôfago sugerem que na acalásia idiopática prevalece a lesão da inervação inibitória e na doença de Chagas as lesões comprometem a inervação inibitória e também a excitatória (Tabela 1). Em sendo esta hipótese verdadeira, a pressão do EIE deveria ser alta na acalásia idiopática e normal ou baixa na doença de Chagas ${ }^{(27)}$.

Vários estudos têm demonstrado que a pressão do EIE na acalásia idiopática está aumentada ${ }^{(6,10,20,29,31)}$. Na doença de Chagas diferentes trabalhos descrevem resultados em que a pressão do EIE está diminuída ${ }^{(10,38)}$ normal $^{(7,15,26,31)}$ ou aumentada ${ }^{(34,40)}$. As evidências de que na acalásia idiopática a inervação excitatória do EIE está íntegra ou só parcialmente comprometida estão na observação de que há na doença hipersensibilidade do EIE à gastrina ${ }^{(6,29)}$, de queda de pressão quando da injeção de toxina botulínica no esfíncter ${ }^{(39)}$, da manutenção das respostas ao edrofônio ${ }^{(29)}$ e à atropina ${ }^{(29)}$, e predomínio da inervação $\alpha$-adrenérgica, em detrimento da inervação $\beta$-adrenérgica ${ }^{(5)}$. Também são encontradas redução do número de fibras com polipeptídio intestinal vasoativo ${ }^{(3,41)}$ e perda funcional dos receptores D2 da dopamina (inibitórios), com manutenção dos receptores D1 (excitatórios) $^{(41)}$.

$\mathrm{Na}$ doença de Chagas ocorre hiposensibilidade do EIE à gastrina ${ }^{(38)}$, predomínio da inervação $\alpha$-adrenérgica em relação à inervação $\beta$-adrenérgica ${ }^{(21)}$, resposta parcial à atropina ${ }^{(9)}$ e à toxina botulínica ${ }^{(4)}$. Nas duas formas de acalásia está demonstrada a perda da inervação não-adrenérgica não-colinérgica inibitória do esôfago ${ }^{(33)^{*}}$.

O controle da pressão do EIE é dado pela ação própria da musculatura, o componente mais importante, a inervação colinérgica ${ }^{(27)}$, o efeito de outros neurotransmissores ${ }^{(41)}$ e a ação de hormônios ${ }^{(25)}$. A participação de hormônios é duvidosa e, caso exista, é de pequena importância. No caso da acalásia a gastrina agiria na inervação colinérgica excitatória que, sem o antagonismo da inervação inibitória, exibe resposta aumentada ${ }^{(6)}$. No caso da doença de Chagas, a resposta está diminuída ${ }^{(38)}$, o que reforça a idéia de comprometimento da inervação excitatória, embora os níveis de gastrina circulantes estejam aumentados $^{(42,43)}$, o que não ocorre na acalásia idiopática ${ }^{(6)}$. Com a colecistocinina, que no esôfago normal provoca diminuição da pressão por agir na inervação inibitória, na acalásia provoca-se o efeito de grande aumento de pressão ${ }^{(17)}$, conseqüente à ação direta na musculatura do esfíncter.

TABELA 1 - Resultados de estudos realizados em pacientes com acalásia idiopática e doença de Chagas

\begin{tabular}{|c|c|c|}
\hline & \multicolumn{2}{|c|}{ Esfíncter inferior do esôfago } \\
\hline & Doença de Chagas & Acalásia idiopática \\
\hline Ação da gastrina & Hipossensibilidade $^{(38)}$ & Hipersensibilidade ${ }^{(6,29)}$ \\
\hline Inervação inibitória & Ausente $^{(30)}$ & Ausente $^{(3,33,41)}$ \\
\hline Inervação excitatória & Ausente $^{(9)}$ & Presente $^{(29)}$ \\
\hline Receptores $\alpha$-adrenérgicos & Predomínio ${ }^{(21)}$ & Predomínio $^{(5)}$ \\
\hline Fibras com polipeptídio intestinal vasoativo & $?$ & Diminuídas $^{(3)}$ \\
\hline Receptores D2 da dopamina & $?$ & Diminuídos ${ }^{(41)}$ \\
\hline Pressão basal & Normal/Aumentada ${ }^{(15,31,34,40)}$ & Aumentada $^{(6,10,29,31)}$ \\
\hline Resposta à toxina botulínica & Redução de pressão $(23 \%)^{(4)}$ & Redução de pressão $(32 \% \text { a } 45 \%)^{(39)}$ \\
\hline Resposta ao edrofônio & $?$ & Presente ${ }^{(29)}$ \\
\hline Resposta à atropina & $\operatorname{Parcial}^{(9)}$ & Presente ${ }^{(29)}$ \\
\hline Gastrina circulante & Aumentada $^{(42,43)}$ & Normal $^{(6)}$ \\
\hline Anticorpos anti M2 & Presente ${ }^{(22)}$ & Ausente $^{(22)}$ \\
\hline
\end{tabular}

$?=$ sem resultados

" Oliveira RB et al. - Dados não publicados. 
Anticorpos contra receptores muscarínicos M2 de acetilcolina são mais freqüentes em pacientes chagásicos com acalásia do que em pacientes com acalásia idiopática. Estes anticorpos aumentam in vitro o tônus da musculatura de $\mathrm{EIE}^{(22)}$.

Não foram encontradas diferenças entre pacientes com acalásia idiopática e aqueles com doença de Chagas na amplitude das contrações esofágicas ${ }^{(1,15)}$, destacando-se que nas duas doenças ela está diminuída em relação a pessoas normais ${ }^{(15)}$, e na amplitude das contrações em faringe, neste local sem diferença com pessoas normais $^{(2)}$. A proporção de pacientes com contrações de baixa amplitude (inferior a $37 \mathrm{~mm} \mathrm{Hg}$ ) é maior nos pacientes com doença de Chagas do que naqueles com acalásia idiopática ${ }^{(13)}$. Trabalhos sobre a complacência esofágica também encontraram resultados semelhantes nas duas doenças, ou seja, aumento da complacência ${ }^{(19,24)}$. A duração da contração esofágica é maior nos pacientes com acalásia idiopática do que nos pacientes com doença de Chagas $^{(1,15)}$ e deglutições seguidas de falha de contração ${ }^{(15)}$, bem como o número de pacientes com falhas de contração ${ }^{(1)}$, são maiores entre os pacientes com doença de Chagas do que entre os pacientes com acalásia idiopática (Tabela 2).
No estudo do esfíncter superior do esôfago foram encontradas pressões semelhantes nas duas formas de acalásia e em pessoas normais em um estudo ${ }^{(14)}$ e pressão baixa na doença de Chagas em outro $^{(2)}$. Na pessoa normal a distensão do esôfago com ar faz a pressão do esfíncter superior do esôfago cair e o ar ser eliminado(32). Em pacientes com acalásia a pressão sobe e o ar é retido, o que pode provocar grande distensão do esôfago e dificuldades respiratórias, com vários casos descritos em pacientes com acalásia idiopática ${ }^{(32)}$, não sendo do nosso conhecimento a existência de casos semelhantes em pacientes com megaesôfago provocado pela doença de Chagas. A amplitude de contração em parte proximal do esôfago, poucos centímetros abaixo do esfíncter superior, é menor nos pacientes com acalásia idiopática do que em controles ${ }^{(16,18)}$, fato não observado em pacientes com doença de Chagas ${ }^{(16)}$.

Estas possíveis diferenças nas lesões presentes nas duas doenças não têm grande influência na apresentação clínica. Observações não controladas sugerem que na acalásia idiopática os sintomas são mais intensos e evoluem com maior rapidez do que na doença de Chagas, mas a confirmação desta hipótese aguarda estudos que visem o seu esclarecimento. Como as doenças têm semelhantes alterações motoras

TABELA 2 - Diferenças entre acalásia idiopática (AI) e aquela conseqüente à doença de Chagas (DC) descritas em trabalhos que compararam as duas doenças

\begin{tabular}{|c|c|c|c|}
\hline & Controles & DC & AI \\
\hline Pressão $\operatorname{EIE}^{(31)}(\mathrm{mm} \mathrm{Hg})$ & $17,9 \pm 7,0$ & $23,5 \pm 12,1$ & $33,3 \pm 13,6^{\mathrm{a}, \mathrm{b}}$ \\
\hline Pressão $\operatorname{EIE}^{(15)}(\mathrm{mm} \mathrm{Hg})$ & $31,2 \pm 11,8$ & $26,0 \pm 12,9$ & $38,7 \pm 14,5^{\mathrm{b}}$ \\
\hline Pressão $\operatorname{EIE}^{(10)}(\mathrm{mm} \mathrm{Hg})$ & $24,9 \pm 10,2$ & $17,8 \pm 9,7^{\mathrm{a}}$ & $40,7 \pm 17,8^{\mathrm{a}, \mathrm{b}}$ \\
\hline \multicolumn{4}{|l|}{ CONTRAÇÕES ${ }^{(15)}$} \\
\hline Amplitude (mm Hg) & $109,1 \pm 44,7$ & $49,3 \pm 38,1^{\mathrm{a}}$ & $37,7 \pm 11,4^{\mathrm{a}}$ \\
\hline Duração (segundos) & $4,1 \pm 1,3$ & $4,6 \pm 1,4$ & $5,8 \pm 1,7^{\mathrm{a}, \mathrm{b}}$ \\
\hline Síncronas (\%) & 2 & $43^{\mathrm{a}}$ & $88^{\mathrm{a}, \mathrm{b}}$ \\
\hline Falhas (\% deglutições) & 2 & $11^{\mathrm{a}}$ & $1^{\mathrm{b}}$ \\
\hline \multicolumn{4}{|l|}{ CONTRAÇÕES $^{(1)}$} \\
\hline Amplitude (mm Hg) & - & $24,6 \pm 11,7$ & $27,4 \pm 10,6$ \\
\hline Duração (segundos) & - & $5,0 \pm 2,0$ & $7,3 \pm 6,4^{b}$ \\
\hline Falhas (\% pacientes) & - & 50 & $26^{\mathrm{b}}$ \\
\hline \multicolumn{4}{|l|}{ CONTRAÇÕES PROXIMAL ${ }^{(16)}$} \\
\hline Amplitude (mm Hg) & $103,8 \pm 41,3$ & $94,3 \pm 44,9$ & $69,9 \pm 49,5^{\mathrm{a}}$ \\
\hline Duração (segundos) & $2,7 \pm 0,8$ & $2,7 \pm 1,4$ & $2,6 \pm 0,8$ \\
\hline Pressão $\operatorname{ESE}^{(14)}(\mathrm{mm} \mathrm{Hg})$ & $113,0 \pm 46,0$ & $126,9 \pm 62,1$ & $114,1 \pm 38,3$ \\
\hline Pressão $\operatorname{ESE}^{(2)}(\mathrm{mm} \mathrm{Hg})$ & $59,5 \pm 23,7$ & $34,4 \pm 18,2^{\mathrm{a}}$ & $45,7 \pm 27,4$ \\
\hline Amplitude faringe $\mathrm{e}^{(2)}(\mathrm{mm} \mathrm{Hg})$ & $105,3 \pm 38,9$ & $123,5 \pm 42,4$ & $107,9 \pm 33,0$ \\
\hline Contrações de baixa amplitude $(\%)^{(9)}$ & 4 & $46^{\mathrm{a}}$ & $34^{\mathrm{a}}$ \\
\hline Freqüência anticorpos anti M2 $(\%)^{(22)}$ & 0 & $84^{\mathrm{a}}$ & $28^{\mathrm{a}, \mathrm{b}}$ \\
\hline
\end{tabular}

Média \pm DP EIE = esfíncter inferior do esôfago; ESE = esfíncter superior do esôfago;

${ }^{\text {a }} P<0,05$ vs controles $\quad{ }^{\text {b }} P<0,05$ vs DC 
no EIE e corpo do esôfago, as possibilidades de tratamento são as mesmas, a escolha da melhor opção para cada paciente na dependência da apresentação clínica e radiológica e da experiência do serviço médico que vai realizar a terapêutica. Trabalho recente, que comparou as diferentes opções de tratamento em pacientes com acalásia idiopática (toxina botulínica, dilatação pneumática e esofagomiotomia laparoscópica), demonstrou que o melhor custo-benefício relacionado à melhora da qualidade de vida ocorre com a dilatação pneumática, considerando-se os primeiros 5 anos após o tratamento ${ }^{(35)}$. Em conclusão, embora a apresentação clínica e o tratamento da acalásia idiopática e da acalásia provocada pela doença de Chagas sejam semelhantes, os trabalhos que estudaram as conseqüências do comprometimento do plexo mientérico do esôfago sugerem que entre as duas doenças há diferenças neste comprometimento.

Dantas RO. Comparison between idiopathic achalasia and achalasia caused by Chagas' disease: a review about the pathophysiology of the diseases. Arq Gastroenterol 2003;40(2):126-130.

ABSTRACT - Background - Although idiopathic achalasia and achalasia caused by Chagas' disease have the same clinical manifestations and treatment, both with destruction of the esophageal myenteric plexus, it is possible that there are differences in the alterations of esophageal motility between the two diseases, caused by different grades of impairment of the excitatory and inhibitory esophageal neurons. Aims - We performed a review of papers with results about the pathophysiology and esophageal motility alterations in idiopathic achalasia and Chagas' disease. Date sources - We reviewed papers which included data about the characteristics of idiopathic achalasia and Chagas' disease. Data synthesis - Impairment of inhibitory esophageal neurons was shown in the two diseases. The results of the studies of the effects of atropine, edrophonium and botulin toxin suggested that the excitatory innervation is more intensely impaired in Chagas' disease than in idiopathic achalasia, explaining the increase in the lower esophageal sphincter pressure found in achalasia. The patients with Chagas' disease have more circulating muscarinic cholinergic receptor M2 autoantibodies than patient with idiopathic achalasia. The duration of the contractions in the esophageal body is longer in idiophatic achalasia than in Chagas' disease. Conclusions - The papers that studied Chagas' disease and idiopathic achalasia, mainly those which studied both diseases with the same methods, suggested that there are different grades of esophageal involvement by the two diseases, mainly the most important involvement of excitatory innervation in Chagas' disease than in idiopathic achalasia.

HEADINGS - Esophageal achalasia. Chagas disease. Esophageal motility disorders.

\section{REFERÊNCIAS BIBLIOGRÁFICAS}

1. Abrahão Jr LJ, Lemme EMO, Domingues GR, Silva Junior JF, Ribeiro VCM. Manometric study of esophageal body in idiopathic and Chagas' disease related achalasia - correlation with the degree of megaesophagus. In: Pinotti HW, Cecconello I, Felix VN, Oliveira MA, editors. Recent advances in diseases of the esophagus. Bologna: Monduzzi; 2001. p 227-33.

2. Abrahão Jr LJ, Lemme EMO, Domingues GR, Val FR, Silva LFD. Uppe esophageal sphincter and pharyngeal function in idiopathic and Chagas' disease related achalasia. In: Pinotti HW, Cecconello I, Felix VN, Oliveira MA, editors. Recent advances in diseases of the esophagus. Bologna: Monduzzi; 2001. p 235-40.

3. Aggestrup S, Uddman P, Sundler F, Fahrenkrug J, Hakanson R, Sorensen HR, Hambraeus G. Lack of vasoactive intestinal polypeptide nerves in esophageal achalasia. Gastroenterology 1983;84:924-7.

4. Brant C, Moraes-Filho JPP, Siqueira E, Nasi A, Libera E Jr, Morais M, Rohr M, Macedo EP, Alonso G, Ferrari AP. Intrasphincteric botulinum toxin injection in the treatment of chagasic achalasia. Dis Esophagus 2003;16:33-8.

5. Bruch HP, Schmidt E, Laven R, Kujath J. Störung der adrenergen Innervation bei Achalasie. Leber Magen Darm 1978;8:17-20.

6. Cohen S, Lipshutz W, Hughes W. Role of gastrin supersensitivity in the pathogenesis of lower esophageal sphincter hypertension in achalasia. J Clin Invest 1971;50:1241-7.

7. Csendes A, Strauszer T, Uribe P. Alterations in normal esophageal motility in patients with Chagas' disease. Am J Dig Dis 1975;20:437-42.

8. Dantas RO. Idiopathic achalasia and chagasic megaesophagus. J Clin Gastroenterol 1988;10:13-5.

9. Dantas RO, Godoy RA, Oliveira RB, Meneghelli UG, Troncon LEA. Effect of isosorbide dinitrate and atropine on the lower esophageal sphincter pressure in chagasic patients. Acta Physiol Pharmacol Latinoam 1988;38:151-8.
10. Dantas RO, Godoy RA, Oliveira RB, Meneghelli UG. Troncon LEA. Lower esophageal sphincter pressure in Chagas' disease. Dig Dis Sci 1990;35:508-12.

11. Dantas RO. Dysphagia in patients with Chagas' disease. Dysphagia 1998;13:53-7.

12. Dantas RO, Deghaide NHS, Donadi EA. Esophageal manometric and radiologic findings in asymptomatic subjects with Chagas' disease. J Clin Gastroenterol $1999 ; 28: 245-8$

13. Dantas RO. Hipocontratilidade do esôfago em pacientes com doença de Chagas e pacientes com acalásia idiopática. Arq Gastroenterol 2000;37:35-41.

14. Dantas RO. Upper esophageal sphincter pressure in patients with Chagas' disease and primary achalasia. Braz J Med Biol Res 2000;33:545-51.

15. Dantas RO, Deghaide NHS, Donadi EA. Esophageal motility of patients with Chagas' disease and idiopathic achalasia. Dig Dis Sci 2001;46:1200-6.

16. Dantas RO, Aprile LRO, Aben-Athar CG, Miranda ALM. Esophageal striated muscle contractions in patients with Chagas' disease and idiopathic achalasia. Braz J Med Biol Res 2002;35:677-83.

17. Dodds WJ, Dent J, Hogan WJ, Patel GK, Toouli J, Arndorfer RC. Paradoxical lower esophageal sphincter contraction induced by cholecystokinin-octapeptide in patients with achalasia. Gastroenterology 1981;80:327-33.

18. Dunaway PM, Maydonovitch CL, Wong RKH. Characterization of esophageal striated muscle in patients with achalasia. Dig Dis Sci 2000;45:285-8.

19. Ejima FH, Dantas RO, Simões MV, Marin Neto JA, Meneghelli UG. Intraesophageal balloon distension test in Chagas' disease patients with noncardiac chest pain. Dig Dis Sci 1998;43:2567-71.

20. Feldman M. Esophageal achalasia syndromes. Am J Med Sci 1988;295:60-81.

21. Fonseca DN. Avaliação da inervação adrenérgica do esfíncter inferior do esôfago em megaesôfago chagásico [tese]. Ribeirão Preto: Faculdade de Medicina de Ribeirão Preto da Universidade de São Paulo; 1999.

22. Goin JC, Sterin-Borda L, Bilder CR, Varrica LM, Iantorno G, Rios MC, Borda E. Functional implications of circulating muscarinic cholinergic receptor autoantibodies in chagasic patients with achalasia. Gastroenterology 1999; 117:798-805. 
23. Goldblum JR, Whyte RI, Orringer MB, Appelman HD. Achalasia. A morphologic study of 42 resected specimens. Am J Surg Pathol 1994;18:327-37.

24. Gonzales M, Mearin F, Vasconez C, Armengol JR, Malagelada JR. Oesophageal tone in patients with achalasia. Gut 1997;41:291-6.

25. Goyal RK, Sivarao DV. Functional anatomy and physiology of swallowing and esophageal motility. In: Castell DO, Richter JE, editors. The esophagus. $3^{\text {th }}$ ed. Philadelphia: Lippincott Williams \& Wilkins; 1999. p. 1-31.

26. Heitmann P, Spinoza J. Oesophageal manometrics studies in patients with chronic Chagas' disease and megacolon. Gut 1969;10:848-51.

27. Hirano I. Pathophysiology of achalasia. Curr Gastroenterol Rep 1999;1:198-202

28. Hirano I, Tatum RP, Shi G, Sang Q, Joehl RJ, Kahrilas PJ. Manometric heterogeneity in patients with idiopathic achalasia. Gastroenterology 2001;120:789-98.

29. Holloway RH, Dodds WJ, Helm JF, Hogan WJ, Dent J, Arndorfer RC. Integrity of cholinergic innervation to the lower esophageal sphincter in achalasia. Gastroenterology 1986;90:924-9.

30. Köberle F. Chagas' disease and Chagas' syndrome: the pathology of American trypanosomiasis. Adv Parasitol 1968;6:63-116.

31. Lemme EMO, Domingues GR, Pereira VL, Firman CG, Pantoja J. Lower esophageal sphincter pressure in idiopathic achalasia and Chagas' disease - related achalasia. Dis Esophagus 2001;14:232-4.

32. Massey BT, Hogan WJ, Dodds WJ, Dantas RO. Alterations of the upper esophageal sphincter belch reflex in patients with achalasia. Gastroenterology 1992;103:1574-9.

33. Mearin F, Mourelle M, Guarner F, Salas A, Riveros-Moreno V, Moncada S, Malagelada JR. Patients with achalasia lack nitric oxide synthase in the gastrooesophageal junction. Eur J Clin Invest 1993;23:724-8.

34. Moraes-Filho JPP, Kohatsu OS, Bettarello A. Pressão basal do esfíncter inferior do esôfago na doença de Chagas: megaesôfago e forma indeterminada. Rev Assoc Méd Bras 1986;32:51-3.
35. O'Connor JB, Singer ME, Imperiale TF, Vaezi MF, Richter JE. The cost-effectiveness of treatment strategies for achalasia. Dig Dis Sci 2002;47:1516-25.

36. Oliveira RB, Rezende-Filho J, Dantas RO, Iazigi N. The spectrum of esophageal motor disorders in Chagas' disease. Am J Gastroenterol 1995;90:1119-24.

37. Oliveira RB, Troncon LEA, Dantas RO, Meneghelli UG. Gastrointestinal manifestations of Chagas' disease. Am J Gastroenterol 1998;93:884-9.

38. Padovan W, Godoy RA, Dantas RO, Meneghelli UG, Oliveira RB, Troncon LEA. Lower oesophageal sphincter response to pentagastrin in chagasic patients with megaoesophagus and megacolon. Gut 1980,21:85-90.

39. Pasricha PJ, Raí R, Ravich WJ, Hendrix TR, Kalloo AN. Botulin toxin for achalasia: long-term outcome and predictors of response. Gastroenterology 1996;110:1410-5.

40. Paula-Costa MD, Rezende JM. Pressão basal do esfíncter inferior do esôfago no meagesôfago chagásico. Rev Assoc Méd Bras 1978;24:269-72.

41. Sigala S, Missale G, Missale C, Villanacci V, Cestari R, Grigolato PG, Lojacono L, Spano PF. Different neurotansmitter systems are involved in the development of esophageal achalasia. Life Sci 1995;56:1311-20.

42. Troncon LEA, Oliveira RB, Meneghelli UG, Dantas RO, Godoy RA. Fasting and food-stimulated plasma gastrin levels in chronic Chagas' disease. Digestion 1984;29:171-6.

43. Troncon LEA, Oliveira RB, Meneghelli UG, Dantas RO, Godoy RA. Plasma gastrin and gastric acid responses to insulin hypoglycemia in Chagas' disease. Braz J Med Biol Res 1985;18:273-8.

44. Wong RKH, Maydonovitch CL. Achalasia. In: Castell DO, Richter JE, editoes. The esophagus. $3^{\text {th }}$ ed. Philadelphia: Lippincott Williams \& Wilkins; 1999. p 185-213.

Recebido em 25/9/2002. Aprovado em 19/12/2002. 